\title{
Adenovirus-expressing TLR5/TLR5 Agonist Nanoformulation M-VM3
}

\author{
National Cancer Institute
}

\section{Source}

National Cancer Institute. Adenovirus-expressing TLR5/T LR5 Agonist Nanoformulation

M-VM3. NCI Thesaurus. Code C131825.

A nanoparticle-based formulation containing a recombinant non-replicating adenovirus (Ad) encoding toll-like receptor 5 (TLR5) and its specific lig and protein 502S, with potential antineoplastic and immunomodulating activities. Upon administration, the Ad preferentially and specifically infects cells expressing the Coxsackievirus and adenovirus receptor (CAR), which is highly expressed in certain human tumors, and expresses both TLR5 and a specific agonistic lig and in the same cell. 502S binds to and activates TLR5, thereby allowing for continuous TLR5 signaling. This stimulates dendritic cells (DCs), monocytes, macrophages and the nuclear factor-kappa B (NF-kappaB) signaling cascade. This activation results in the production of pro-inflammatory cytokines, including interferon alpha, tumor necrosis factor-alpha and the interleukins (IL), IL-1 beta, -6 and -12 . This may induce a T helper cell-1 (Th1) immune response and activate a cytotoxic T-lymphocyte (CTL) response ag ainst tumor associated antigens (TAAs). TLR5, a member of the TLR family, plays a key role in the activation of innate immunity. 